Supporting informations for the manuscript entitled:

\title{
Oriented and nanostructured polycarbonate substrates for the orientation of conjugated molecular materials and gold nanoparticles
}

\author{
Martin Brinkmann $*^{1}$, Sirapat Pratontep ${ }^{3}$, Christian Chaumont ${ }^{2}$, and Jean-Claude \\ Wittmann $^{1}$
}

(1) Institut Charles Sadron, 6, rue Boussingault, 67083 Strasbourg, France

(2) Ecole Européenne de Chimie des Polymères et Matériaux, 25 rue Becquerel, 67087 Strasbourg Cedex 2, France

(3) National Nanotechnology Center (NANOTEC), National Science and Technology Development Agency (NSTDA), 111 Thailand Science Park, Paholyothin Rd., Klong 1, Klong Luang, Patumthani 12120, Thailand 
* Corresponding author: Brinkman@ics.u-strasbg.fr

Figure S1. Selected Area electron Diffraction (SAED) of a pentacene thin film (40nm) deposited at $T_{s}=29^{\circ} \mathrm{C}$ on oriented and nanostructured PC. The main reflections were indexed by using the triclinic structure by Campbell et al. (see text) ${ }^{[22]}$.

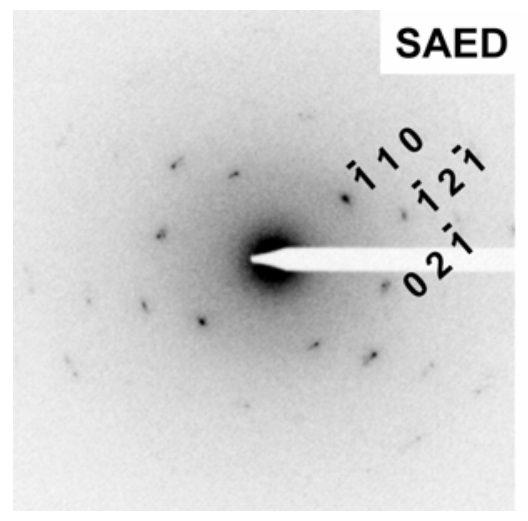

Figure S2. 40nm-thick pentacene films on PC substrates observed under crossed polarizers and showing the existence of pentacene domains with different orientations 

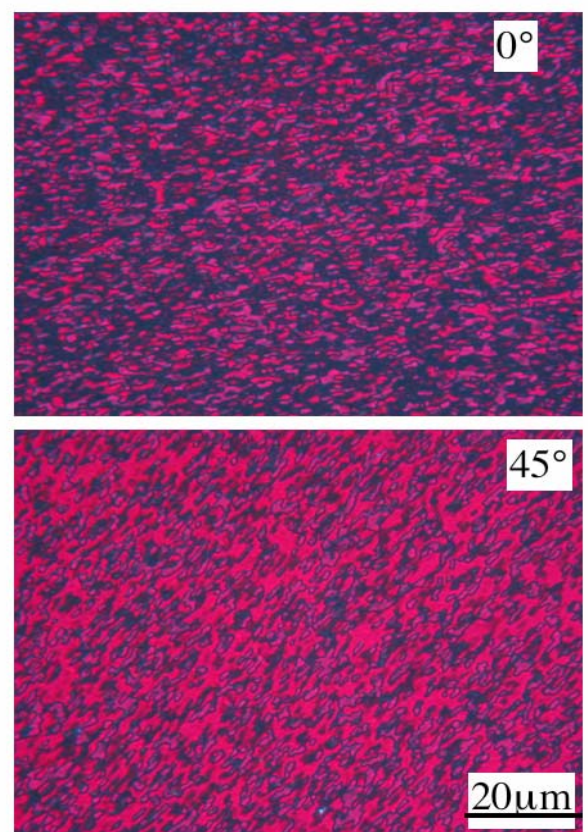

Figure S3. Topography of an oriented film of coronene (50nm) grown at $T_{s}=29^{\circ} \mathrm{C}$ on a PC substrate. The inset corresponds to the ED pattern in proper relative orientation to the AFM morphology.

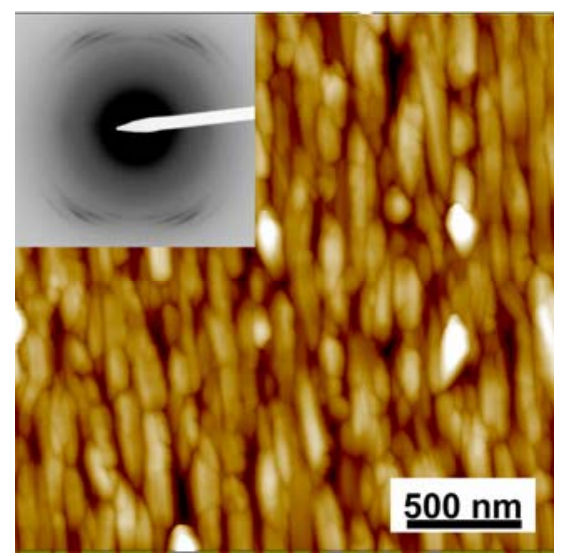


Figure S4 : Surface topography of a thin isotactic polystyrene (iPS) thin film prepared by combining rubbing and surface crystallization. The processing conditions for the rubbing and the solvent induced surface crystallization of iPS are identical to those used in the case of bisphenol A polycarbonate (see text).

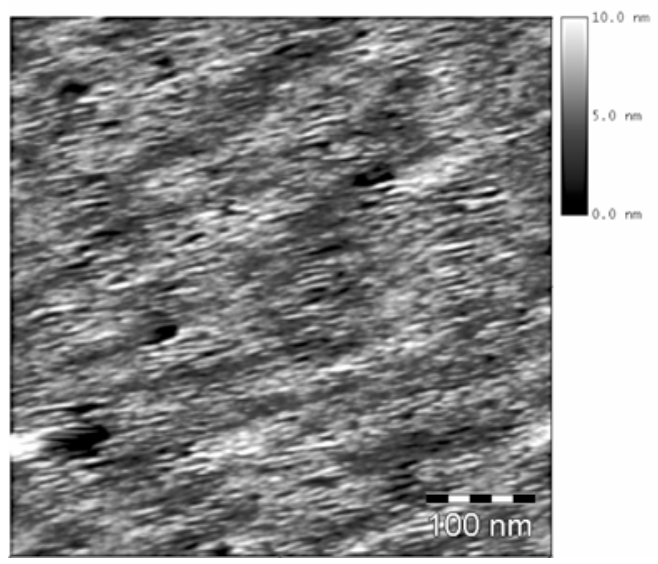

